ÉGYPTE monde arabe

\section{Égypte/Monde arabe}

$11 \mid 1992$

À propos de la nationalité

\title{
Les Karaïtes. Une communauté cairote à l'heure de l'État-nation
}

Frédéric Abécassis et Jean-François Faü

\section{CpenEdition}

\section{Journals}

Édition électronique

URL : https://journals.openedition.org/ema/307

DOI : 10.4000/ema.307

ISSN : 2090-7273

\section{Éditeur}

CEDEJ - Centre d'études et de documentation économiques juridiques et sociales

\section{Édition imprimée}

Date de publication : 30 septembre 1992

Pagination : 47-58

ISSN : 1110-5097

\section{Référence électronique}

Frédéric Abécassis et Jean-François Faü, « Les Karaïtes. Une communauté cairote à l'heure de l'Étatnation », Égypte/Monde arabe [En ligne], 11 | 1992, mis en ligne le 08 juillet 2008, consulté le 07 juillet 2022. URL : http://journals.openedition.org/ema/307 ; DOI : https://doi.org/10.4000/ema.307

Ce document a été généré automatiquement le 7 juillet 2022.

Tous droits réservés 


\title{
Les Karaïtes. Une communauté cairote à l'heure de l'État-nation
}

\author{
Frédéric Abécassis et Jean-François Faü
}

1 Le 21 décembre 1910, le président de la société Ahavat du Caire présentait, dans une lettre en français adressée à son homologue de Cologne, la population juive de la capitale égyptienne, divisée en trois communautés: sépharadim, ashkénazim et karaïtes. Souvent cités par les voyageurs européens, omniprésents dans les descriptions du quartier juif Haret al-Yehudi, mais se dérobant au regard étranger, les karaïtes semblaient faire corps avec leur rue et leur ville, du moins jusqu'au XIX ${ }^{e}$ siècle.

2 La doctrine karaïte a vu le jour en Mésopotamie en 760 . Son fondateur, un rabbin nommé Anan Ben David, d'une famille d'exilarques, rejeta toute référence au Talmud. Se basant uniquement sur l'enseignement de la Torah, il dénia tout pouvoir aux savants talmudistes, les gaonim. Anan accusait les institutions rabbiniques de perdre l'esprit des Ecrits dans le dédale de trop nombreuses lois. Il proclama le droit et le devoir, pour tout juif, de pouvoir interpréter la Torah selon son propre point de vue, sans aucune référence à une quelconque interprétation officielle du rabbinat ${ }^{1}$. Anan rallumait la vieille querelle qui avait opposé Esséniens et Pharisiens au premier siècle de notre ère, et provoquait une coupure à l'intérieur du judaïsme. Ainsi le mode de vie karaïte, très vite considéré comme hérétique par le judaïsme orthodoxe, se distinguait par une application beaucoup plus rigoureuse de la Loi, Par exemple, les karaïtes ne laissaient pas le feu se consumer pendant le Shabat, à fa différence des juifs traditionnels, pour qui l'interdit porte seulement sur l'acte (allumer le feu), et non sur le fait (laisser brûler une flamme). De même ils ignoraient, tout comme les Falashas, la fête de Hanouka (la fête des lumières), qui est post-biblique. La communauté en rupture éprouvait également le besoin d'affirmer de nouvelles pratiques rituelles. Les karaïtes se déchaussaient avant de pénétrer dans la synagogue et se prosternaient devant la Teva (le Tabernacle), pour prier en arabe le Dieu d'Israël. La polygamie, quoique peu pratiquée, ne leur fut jamais interdite. Popularisée par des écrits de qualité, la doctrine karaïte s'étendit parmi les communautés Juives d'Irak et de Perse, puis gagna les bords de la Mer noire. Elle se propagea ensuite en Palestine au xe siècle, et arriva en Égypte. 
Un certain nombre de familles persanes, dont les Tustarie et les Firuz, originaires de Tabriz, vinrent s'installer à al-Fustat dès le $\mathrm{xl}^{\mathrm{e}}$ siècle. Plusieurs voyageurs mentionnèrent, au fil des siècles, la présence de ces marginaux du judaïsme, de Benjamin de Tudèle au XII ${ }^{e}$ siècle à Léon de Modène au XviII ${ }^{e}$ siècle. Les documents de la Guenizah du Caire les citent, avec les Samaritains, et attestent même de cas de mariages entre karaïtes et rabbiniques, familles divisées par le rite, mais unies par le même métier : le négoce.

$3 C^{\prime}$ 'est au XIII ${ }^{\text {e }}$ siècle que les karaïtes s'installèrent dans le Haret al-Yehudi, au Muski, où ils occupèrent une rue réservée, chari' al-Karayin. Estimée à environ 3000 personnes en 1798, la communauté se diversifia au $\mathrm{xIX}^{\mathrm{e}}$ siècle sous la double pression démographique et sociale.

\section{Une communauté de l'Empire ottoman}

4 Un flux d'immigration toucha, à partir de la moitié du xix ${ }^{e}$ siècle, aussi bien la communauté rabbinique que karaïte. Cette dernière se trouva gonflée, en 1860 , de réfugiés fuyant les massacres de Damas. La communauté karaïte de cette ville disparut cette même année, ses membres ayant pris le chemin du Caire ou d'Istanbul. L'intégration des nouveaux arrivants fut d'autant plus facile qu'ils étaient tous arabophones et artisans, ce qui leur permit de se fondre sans peine dans le cadre social déjà existant. Beaucoup de karaïtes exerçaient alors des professions d'artisanat spécialisé, comme menuisier, tailleur ou graveur. Leur condition de vie restait toutefois modeste. La structure familiale, les coutumes étaient très proches de celles des autres communautés populaires égyptiennes. Les hommes portaient la galabeyya, les femmes étaient voilées de la coiffure traditionnelle des juives égyptiennes, la habara, pièce de tissu noir couvrant la tête et nouée dans le dos. Les prénoms étaient arabes - Amin, Mourad ou 'Abd al-'Aziz ; la polygamie était encore pratiquée, puisqu'on en recensait 25 cas en $1904^{2}$. Pour tous, l'arabe était la langue quotidienne, culturelle et cultuelle. 

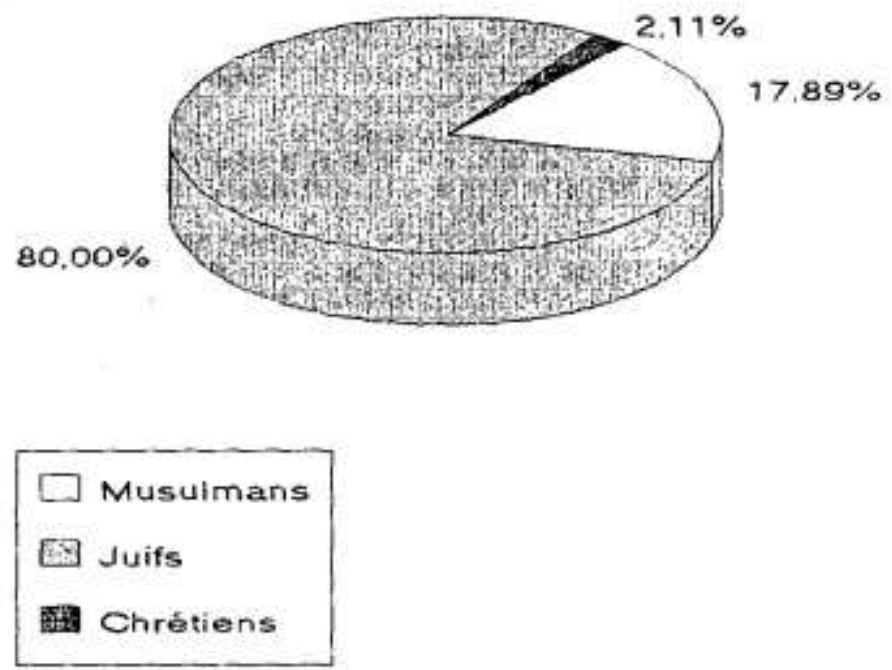

La communauté était dirigée par une petite oligarchie de notables, artisans ou joailliers, dont le niveau social tendait à s'élever au fil des ans. Ils habitaient eux aussi la rue des karaïtes, mais portaient volontiers le tarbouche, méprisaient la galabeyya et ne fréquentaient leur coreligionnaires qu'à la synagogue.

En cette fin du XIX ${ }^{e}$ siècle, deux temples étaient en service. L'un, plus ancien et presque en ruine, construit en soubassement, était connu sous le nom de Bayt-Knesset al-Qadîm et servait de lieu d'asile aux indigents. Une seconde synagogue, datant du XVIII ${ }^{e}$ siècle, se nommait Khan Abu Takieh. Les synagogues karaïtes n'avaient aucune particularité architecturale et ressemblaient à celles des rabbiniques. Toutes deux avaient le même plan basilical. La galerie réservée aux femmes s'étendait à l'étage sur trois côtés. Le mur est comportait la niche de fa Torah, la Teva ; la partie centrale de la nef était occupée par la Teba, le pupitre destiné à la lecture des Ecritures. Une différence, toutefois, dans le mobilier : les synagogues karaïtes ne comportaient ni bancs ni chaises, mais des tapis de prière sur lesquels les orants se prosternaient. Les officiants, bien que refusant toute référence au rabbinat du Caire, portaient soit le titre de rabbi, soit celui de hakam.

Vers 1860, une vingtaine de familles quittèrent le Caire pour s'installer à Alexandrie. Elles s'insérèrent dans les cadres de la communauté séfarade, sauf en ce qui concerne le culte, pour lequel elles continuèrent à faire appel à leur rabbi du Caire. Ce groupe comptait 243 membres en 1947.

La communauté karaïte fut reconnue indépendante de la communauté rabbinique en 1848. Le siège social de la communauté était situé, depuis 1860 , dans un immeuble de la rue des Karaïtes. Le hatti humayoun, promulgué à Istanbul par le Sultan 'Abd al-Magid en 1856, et les deux hautes circulaires des 3 février et 1er avril 1891, réglementaient les tribunaux confessionnels et la compétence des conseils communautaires (ou maglis milli) dans tout l'Empire ottoman. Après un début d'application ponctuelle de ces textes en Égypte, le gouvernement demanda en 1906 à toutes les communautés de présenter leur projet de règlement intérieur pour les approuver et leur donner vigueur de loi 
exécutive. En 1933, seules quatre communautés non musulmanes, dont la communauté karaïte, n'avaient pas présenté le leur. Ainsi, les jugements de leurs tribunaux religieux ou mixtes ne recevaient pas force exécutoire de la part des bureaux administratifs du gouvernement.

Ecole de la communauté karaïte du Caire (Waïly) Répartition des effectifs par religion en 1912-1913

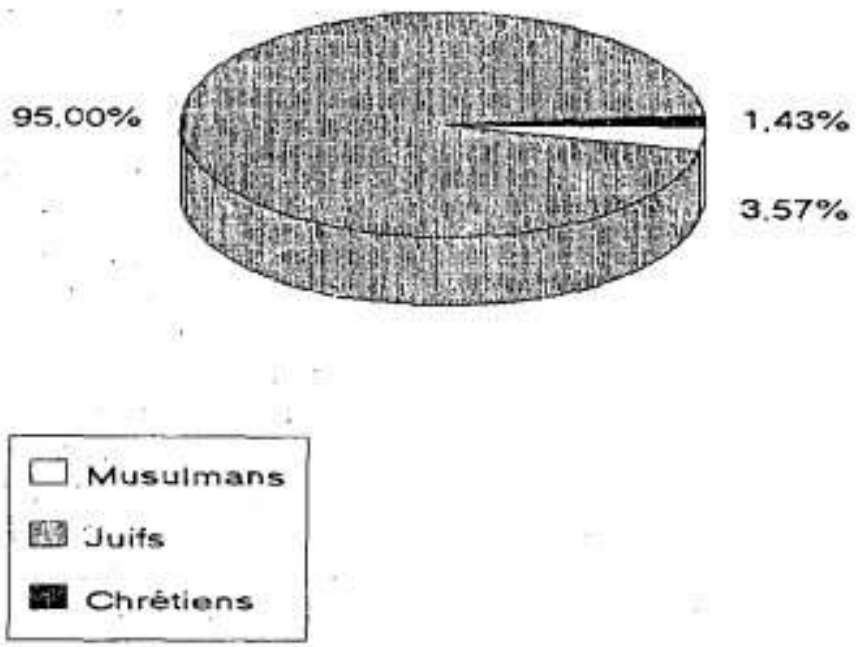

9 Les karaïtes étaient cependant organisés selon le schéma classique d'une communauté. A la base, on trouvait les contribuables, qui payaient le don du culte; le conseil communal (maglis milli) existait depuis 1901. Placé sous la présidence du Grand Rabbin karaïte, il se composait de douze membres tirés au sort, et sa fonction était de juger les procès religieux. Ses membres étaient tous issus de la haute-bourgeoisie, tels Amin Farag ou 'Abd al-Aziz 'Abd al-Wahab. Le conseil communal élisait un président, un viceprésident et un secrétaire général, qui ont toujours été égyptiens. Ainsi, les affaires communautaires semblaient réservées aux seuls Cairotes, sauf pour le hakam. qu'une tradition faisait venir du Caucase. Ce hakam était élu par un conseil général dont les membres étaient ceux du maglis milli, auquel se joignaient vingt-huit notables. Ce conseil avait également le pouvoir de le révoquer. La communauté karaïte obéissait à la loi mosaïque en ce qui concerne les mariages et les divorces. En revanche elle suivait la loi musulmane pour toutes les questions de succession : le testateur ne disposait, que du tiers de la succession, .qu'il pouvait dévoluer à qui bon lui semblait,'et aucun héritier légitime n'était favorisé au détriment .des autres sans leur consentement.

\section{Entre impérialisme et État}

En 1897, un choc ébranla le karaïsme cairote : la fondation d'un établissement de l'Alliance Israélite universelle au Caire. Cette fondation eut lieu à une période où la communauté se trouvait en plein bouleversement. Grâce à l'ouverture du canal de Suez et aux investissements immobiliers, une partie de la petite bourgeoisie du Haret alYehudi était arrivée à atteindre un degré social supérieur, celui de la classe d'affaires. De grandes familles se détachèrent, les Cohen, les Tawil ou les Massaouda, et 
s'installèrent dans le quartier résidentiel de 'Abbasseya. L'Alliance Israélite universelle y ouvrit une école annexé où cette bourgeoisie d'affaires fit inscrire ses enfants, boudant l'école communautaire du Haret al-Yehudi. En 1902, les registres relèvent 16 karaïtes sur 587 élèves, soit $3 \%$ des effectifs. Débuts modestes, certes, mais qui ne doivent pas masquer une volonté de rompre délibérément avec le passé. Celle-ci peut également se lire dans la scolarisation de certains enfants karaïtes dans les écoles des missions religieuses françaises, ou au lycée de la Mission laïque.

Effectifs de la communauté karailte selon la natonalite
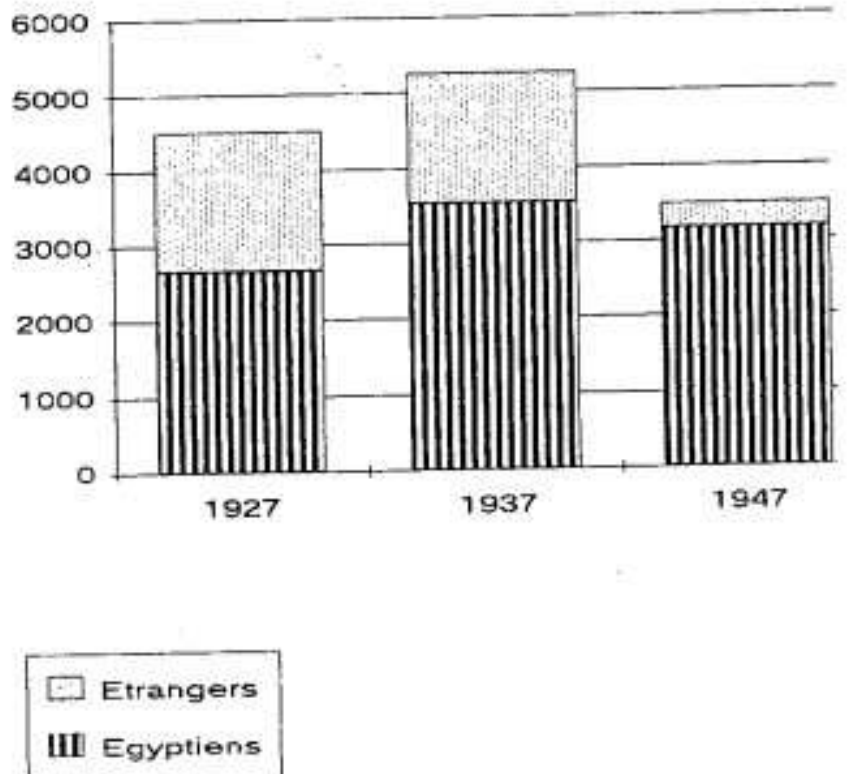

11 A la différence du rabbinat, qui méprisait les karaïtes en tant qu'arabophones et les rejetait comme hérétiques, l'Alliance Israélite universelle, dans sa conception culturelle $\mathrm{du}$ judaïsme, ramenait dans le giron communautaire les juifs orientaux en les francisant. En effet, le programme officiel de l'Alliance laissait une place à l'histoire des karaïtes ${ }^{3}$. Cette politique culturelle constituait la véritable force d'attraction de l'Alliance en cette fin de siècle. Tant d'efforts ne laissèrent pas inactifs ses principaux rivaux. Tout d'abord, première concernée; la communauté karaïte réagit en fondant en 1899 un établissement scolaire. Il se composait de deux écoles, une de garçons, une de filles, installées dans un bâtiment du Muski. Deux cycles scolaires y étaient assurés, un primaire et un préparatoire; la majorité des disciplines y étaient enseignées en arabe. En 1906, l'école recevait un total de 216 élèves, dont un certain nombre de musulmans. En 1910, l'école déménagea, quittant les ruelles du Haret al-Yehudi pour s'installer à Waïly, non loin de 'Abbasseya. Le souhait d'endiguer le succès de l'école de l'Alliance ne fut certainement pas étranger à ce déménagement. Ce transfert eut une conséquence directe sur le fonctionnement de l'école, qui se divisa en deux établissement distincts, un premier pour les garçons, avec un corps professoral de douze enseignants, et le second plus modeste pour les filles, avec trois professeurs. Le fait d'abandonner son environnement d'origine fit perdre à l'école karaïte son .aspect d'école de quartier, qui accueillait, en plus de son public ordinaire, les musulmans du voisinage. Ces derniers ont en effet presque disparu des salles de classe du Waïly. Vingt-huit ans après l'ouverture de cet établissement, en 1927, la communauté comprenait encore $50 \%$ 
d'analphabètes. Ce pourcentage tombe à $40 \%$ en 1937, ce qui reste un taux supérieur à celui de la communauté rabbinique, globalement plus instruite.

Les associations sionistes égyptiennes furent fondées en 1897, en même temps que l'Alliance israélite universelle. Elles recrutèrent surtout dans la communauté ashkénaze, restant sans illusions sur l'engagement des karaïtes : « Les sépharadim et les karaïtes forment un bloc compact, établi depuis de longues années dans notre pays, et sont ainsi restés étrangers à ce que le sionisme a créé depuis les dix dernières années ${ }^{4}$ Les karaïtes, en même temps que les samaritains, posaient un réel problème éthique à ces organisations sionistes, en quête de fondements unitaires pour le judaïsme. Ainsi le 22 février 1922, le Dr Pilpoul, président de la commission de propagande de la Fédération sioniste d'Égypte, déclara (en français), lors d'une soirée organisée parla Fédération : «Il faut supprimer les dénominations ashkénazim. sépharadim et karaïte ; ne doit subsister qu'une seule communauté sous les auspices de l'organisation sioniste. »

En dépit toutefois des efforts déployés, les karaïtes ne portèrent que très peu de crédit à l'idée de la création d'un état juif. La masse, assimilée, vivait et mourait dans son quartier, avec une vision du monde à l'échelle du Muski; tandis que les élites n'avaient de cesse de s'intégrer à une communauté francophone cairote qui, sous l'action de l'Alliance Israélite universelle, se tournait de plus en plus vers l'étranger. Le schéma linguistique correspondait surtout à un choix culturel et politique. Le français représentait le passage obligé de l'émancipation sociale, mais entérinait aussi un clivage avec le reste des habitants de la rue des karaïtes. La communauté karaïte fut affectée par les transformations de la première moitié $\mathrm{du} \mathrm{xx}^{\mathrm{e}}$ siècle selon le même schéma que celui des chrétientés orientales : enrichissement d'une fraction, suivi d'une opération de séduction des missions européennes allant parfois jusqu'au mépris de la culture d'origine.

En 1927, on recensait dans la communauté karaïte 1.848 étrangers, surtout russes et syriens, et 2659 Égyptiens. La loi sur la nationalité de 1926, révisée en 1929, mettait en demeure les anciens sujets ottomans d'opter pour la nationalité de leur choix. Une petite partie de la communauté avait opté en 1937 pour la nationalité égyptienne, mais la plupart des étrangers, qu'avait rejoint une vague d'immigration russe vers la fin des années 1920, avaient sans doute préféré tourner leur civisme vers les consulats européens. La plupart d'entre eux vivaient à 'Abbasseya où, signe de prospérité, une synagogue fut construite en 1927, la Khan 'Abbasseya, après une souscription publique de 12000 LE.

15 C'est à cette époque que le siège de la communauté se déplaça du Muski à 'Abbasseya, tandis que l'autorité religieuse, le hakam, continua de siéger au Haret al-Yehudi. L'organisation de la jeunesse karaïte du Caire, al-gama'iya al-chubban al-isrấiliyyîn alkarâyin bi Misr, dirigée par Ya'qub Farag 'Abd Allah, installait également son siège rue Tur Sinaï à 'Abbasseya. La communauté fondait à cette époque un journal en langue arabe, al-Chubban qui, sous le nom de al-Kalim à partir de 1945, continua de paraître jusqu'en 1957. Elle se dotait ainsi d'un organe de presse culturel et religieux dans la tradition des journaux du début, du siècle. Toute une société s'organisait donc à l'intérieur d'un monde bipolaire, partagé entre 'Abbasseya et Haret al-Yehudi, aveugle aux changements extérieurs.

16 Pourtant, durant la première moitié $\mathrm{du} \mathrm{xx}^{\mathrm{e}}$ siècle, quelques personnalités se détachèrent, s'inscrivant dans une mouvance nationaliste qui prenait de plus en plus 
d'importance. On peut citer Mourad Farag, né en 1837, avocat auprès des juridictions nationales, qui plaida dans l'affaire Kahana en 1902 pour défendre son client de l'accusation de meurtre rituel. Nationaliste, il critiqua, durant sa plaidoirie, les interventions étrangères auprès des cours égyptiennes. D'autres karaïtes connurent un certain succès politique ou culturel, comme le militant communiste Youssef Darwich qui finira par se convertir à l'islam - ou le musicien Dawud Hosni.

La fin de la seconde guerre mondiale vit les événements se précipiter. En 1942, Ya'qub Farag 'Abd Allah, ancien président du cercle de la jeunesse, avait succédé à Liéto Baruch Masawuda à la tête de la communauté. La création de l'État d'Israël et la guerre de 1948 prirent celle-ci au dépourvu. En effet, seule la minorité étrangère s'était préparée à quitter l'Égypte et avait pu prendre quelques dispositions financières. La plupart de ses membres avaient déjà quitté l'Égypte en 1947. Il est vraisemblable que, contrairement à leurs coreligionnaires égyptiens, mal à l'aise dans les structures de plus en plus nettement affirmées de l'État-nation, ils furent séduits par ta loi du retour et la citoyenneté qu'elle leur apporterait. Les émeutes qui firent suite au grand incendie du Caire en 1952 n'épargnèrent pas les juifs et leur firent nourrir beaucoup d'inquiétude sur leur avenir en Égypte. La visite et le discours du général Néguib au, siège de la communauté karaïte, puis à la grande synagogue du Caire le soir du Yom Kippour de 1953, rassurèrent un temps les esprits ${ }^{5}$. Mais l'affaire Lavon, en juillet 1954, marqua la fin de ce rapprochement. Un membre de la communauté karaïte était en effet impliqué dans une affaire d'espionnage au profit d'Israël. La vague d'émigration qui succéda au coup de force de Suez emporta la quasi-totalité d'une communauté profondément traumatisée, qui abandonna presque tous ses biens immobiliers. Beaucoup se fixèrent à Ramla, à proximité de Tel-Aviv, où ils furent rejoints par les membres de la dernière communauté karaïte en pays arabe, celle de Hitt en Irak. Sa'd al-Dîn Ibrahim estimait leur nombre à 150000 personnes à la fin des années $1980^{6}$.

$\mathrm{Ne}$ restèrent au Caire que les vieillards et les indigents. Ils étaient encore une quarantaine en 1979, pris en charge par la Croix-Rouge internationale. Ils ne sont plus que huit de nos jours, habitant toujours le quartier du Muski. Le dernier de leurs représentants garde les archives de la communauté qui, en l'absence de tout classement, demeurent inaccessibles.

Ainsi disparut une communauté millénaire, qui était demeurée davantage cairote qu'égyptienne. Divisée par l'évolution sociale du xix siècle et incapable de se plier à l'engagement au sein d'un État-nation; elle ne put ou ne sut choisir, si ce n'est sous la pression tout à coup intensifiée des événements.

\section{BIBLIOGRAPHIE}

Courbage Youssef et Fargues Philippe, Chrétiens et Juifs dans l'Islam arabe et turc, Fayard, Paris, 1992, 345 p.

Fargeon Maurice, Les Juifs en Égypte, Barbey, Le Caire, 1938, 321 p. 
Goiten S. D., A Mediterranean society, the Jewish communities of the Arab word as portrayed in the documents of the Cairo Gueniza, 4 tomes, The Community, University of California Press, Berkeley, 1971.

Graizel Salomon, A History of the Jews, Jewish Publication Society of America, New-York, 1968, 768 p.

Kramer Gudrun, The Jews in Modern Egypt 1914-1952, Seattle, University of Washington Press, 1989, $319 \mathrm{p}$.

Landau Jacob, Jews in XIX century in Egypt, New-York University Press, Londres, 1969. 354 p.

Lewis Bernard, Juifs en terre d'Islam, Flammarion, Paris. 1984. 258 p.

Sa'd al-Din Ibrahim, Réflexions sur la question des minorités, co-édition Markaz Ibn Khaldoun (Le Caire) et Dar Suad Al-Sabah (Koweit), 1992 (en arabe), 250 p.

Szyszman Simon, « Les Karaïtes d'Égypte et leurs trésors », in Le monde copte n 6, Paris. 1979, p. 37-44.

Szyszman Simon, Le Karaïsme, Lausanne, L'Age d'Homme, 1980, 247 p.

Talgram Rina et Yaniv Braha, « Survey of Jewish visual art in Egypt », in Bulletin of Israeli Academic Center in Cairo n 4, été 1984.

Annuaire des Juifs d'Égypte, Société des éditions historiques Juives d'Égypte, Le Caire, 1942.

Statistique de l'Enseignement en Égypte, Imprimerie nationale, Le Caire, années 1907-1908 et 1912-1913.

\section{NOTES}

1. S. D. Goiten signale que le karaïsme, favorable à un ascétisme rigoureux, condamna, dès son origine, la vie aisée de la diaspora et préconisa une émigration immédiate vers la Palestine. Goiten S. D., t. Il, p. 7 (voir bibliographie ci-dessous).

2. Nassau R., Die Welt n² 20, 1904.

3. Fresco M., Histoire des Israélites, Paris, réédition 1948 (livre officiel du programme de cours moyen de l'Alliance Israélite universelle).

4. Lettre de la société sioniste du Caire à l'Office sioniste de Cologne, 21 décembre 1910, cité par Landau (voir biblio.), p. 327.

5. Cf. Szyszman in Le Monde copie $\mathrm{n}^{\circ} 6$ (voir biblio\}.

6. Cf. Sa'd al-Din, p. 109 (voir biblio).

\section{INDEX}

Mots-clés : Juifs, communauté, israélites 
AUTEURS

FRÉDÉRIC ABÉCASSIS

Centre d'études françaises - Le Caire

JEAN-FRANÇOIS FAÜ

Centre d'études françaises - Le Caire 